\title{
Influence of Electron Beam Energy and Dose on In Situ Electron Microscopy Studies for Direct Correlation between Structure and Properties
}

\author{
Eva Olsson $^{1 *}$
}

1. Department of Physics, Chalmers University of Technology, 41296 Gothenburg, Sweden.

* Corresponding author: eva.olsson@chalmers.se

In situ electron microscopy has the capability of combining high spatial resolution imaging and spectroscopy with local probing of properties, for example electrical and mechanical properties. A knowledge about the correlation between atomic structure and properties provides an understanding of the mechanisms that determine the properties and allows us to predict how to control them and design new material structures with tailored performance.

Based on a concept where we have a scanning tunneling microscope (STM) inserted in scanning electron microscopes (SEM) or transmission electron microscope (TEM) holders [1,2], we have developed methods for different types of in situ experiments where we study electric field, light, mechanical strain and temperature induced effects on material structure and properties with high spatial resolution for imaging, diffraction and spectroscopy $[3,4]$. However, it is crucial to consider and understand the effects of the interaction between the electron beam and the specimen to correctly interpret the results. In this presentation, we will illustrate different manifestations of electron beamsample interaction and also the influence of beam energy and dose on the interaction [3-6].

Organic materials constitute one group of materials where the electron beam can have a strong influence on the structure. Here, we will show data from organic solar cell thin films with information about the effect of electron beam energy and dose [6]. The samples were nanostructured polymer:fullerene bulk heterojunction thin films. It has been shown that it is important that the films maintain the fine scale nanostructure during the different fabrication stages that include thermal anneals at about $100{ }^{\circ} \mathrm{C}$. During in situ microscopy observations using parameters corresponding to the annealing temperatures it was found that the electron beam enhanced the thermal stability of the film structure. The electron beam induced cross linking in the film structure that locked the scale of microstructural features. The rate of cross linking depended on electron beam energy and dose.

Another effect of the electron beam- sample interaction is an increase of temperature at the areas exposed to the electron beam. The temperature increase depends on the thermal conductivity of the sample. Here, we will present experimental data from direct local measurements of the difference specimen temperature with and without electron beam exposure [3].

Additional examples of observations of electron beam-sample interactions and how to optimize the parameters for in situ experiments and high precision spatial data recording will be also presented.

\section{References:}

[1] K. Svensson, Y. Jompol, H. Olin and E. Olsson, "Compact design of a transmission electron microscope-scanning tunneling microscope holder with three-dimensional coarse motion", Rev. Sci. Instrum. 74 (2003) 4045. 
[2] K. Svensson, H. Olin and E. Olsson, ’Nanopipettes for metal transport”, Phys. Rev. Lett. 93 (2004) 145901.

[3] L. de Knoop, M.J. Kuisma, J. Löfgren, K. Lodewijks, M. Thuvander, P. Erhart, A. Dmitriev and E. Olsson, "Electric field controlled reversible order-disorder switching of a metal tip surface", Phys. Rev. Mat. 2 (2018) 085006.

[4] J. Holmér, L.J. Zeng, T. Kanne Nordqvist, J. Nygård, P. Krogstrup, L. De Knoop and E. Olsson, “An STM-SEM setup for characterizing photon and electron induced effects in single photovoltaic nanowires", Nano Energy 53 (2018) 175.

[5] T. Nilsson Pingel, M. Jørgensen, A.B. Yankovich, H. Grönbeck and E. Olsson, "Influence of Atomic Site-Specific Strain on Catalytic Activity of Supported Nanoparticles", Nature Communications 9 (2018), 2722.

[6] O. Bäcke, C. Lindqvist, A.D.D. Mendaza, S. Gustafsson, E. Wang, M.R. Anedrsson, C. Müller, P.M. Kristiansen, E. Olsson, "Enhanced thermal stability of a polymer solar cell blend induced by electron beam irradiation in the transmission electron microscope", Ultramicroscopy 176 (2018) 23.

[7] The author acknowledges funding from the Swedish Research Council, the Swedish Energy Agency, Chalmers Area of Advance Materials Science, Chalmers Excellence Initiative Nano and the Knut and Alice Wallenberg Foundation. The work was performed in part at Chalmers Materials Analysis Laboratory. 